Article

\title{
On the Solutions of a Class of Integral Equations Pertaining to Incomplete $H$-Function and Incomplete $\bar{H}$-Function
}

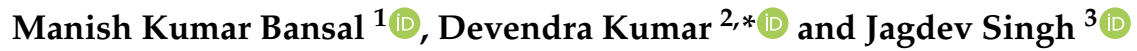 \\ and Kottakkaran Sooppy Nisar ${ }^{4, *}$ \\ 1 Department of Applied Sciences, Government Engineering College, Banswara 327001, Rajasthan, India; \\ bansalmanish443@gmail.com \\ 2 Department of Mathematics, University of Rajasthan, Jaipur 302004, Rajasthan, India \\ 3 Department of Mathematics, JECRC University, Jaipur 303905, Rajasthan, India; \\ jagdevsinghrathore@gmail.com \\ 4 Department of Mathematics, College of Arts and Sciences, Prince Sattam bin Abdulaziz University, \\ Wadi Aldawasir 11991, Saudi Arabia \\ * Correspondence: devendra.maths@gmail.com (D.K.); n.sooppy@psau.edu.sa (K.S.N.)
}

Received: 8 April 2020; Accepted: 11 May 2020; Published: 19 May 2020

check for updates

\begin{abstract}
The main aim of this article is to study the Fredholm-type integral equation involving the incomplete $\mathrm{H}$-function (IHF) and incomplete $\bar{H}$-function in the kernel. Firstly, we solve an integral equation associated with the IHF with the aid of the theory of fractional calculus and Mellin transform. Next, we examine an integral equation pertaining to the incomplete $\bar{H}$-function with the help of theory of fractional calculus and Mellin transform. Further, we indicate some known results by specializing the parameters of IHF and incomplete $\bar{H}$-function. The results computed in this article are very general in nature and capable of giving many new and known results connected with integral equations and their solutions hitherto scattered in the literature. The derived results are very useful in solving various real world problems.
\end{abstract}

Keywords: Fredholm-type integral equations; incomplete $H$-functions; incomplete $\bar{H}$-functions; Mellin transform; Weyl fractional integral

MSC: 45B05; 33C60; 44A20; 33B20; 26A33

\section{Introduction}

Several types of integral equation appear in the fields of engineering and science. They can be used to describe many physical problems. Integral equations have a variety of applications in fields including fluid mechanics, mathematical physics, quantum mechanics, queuing theory, etc. (for details, see [1-11]). Therefore, the study of integral equations and methods for solving them are very useful in science and engineering. Several authors introduced and studied the Fredholm integral equation involving special functions such as Legendre functions, hypergeometric function, and Fox H-function [12-20].

Incomplete special functions have variety of uses in the field of probability theory, communication theory, modeling of groundwater pumping, engineering, science, heat conduction, etc. Very recently, many authors work on these incomplete special functions (for details, see the works of Bansal et al. [21-25], Srivastava et al. [26], Kumar et al. [27], Bansal and Kumar [28], and Chaurasia and Kumar [29]). 
Motivated by the work of Srivastava and Raina [18], we introduce the Fredholm-type integral equation involving the IHF and incomplete $\bar{H}$-function in the kernel.

\section{Preliminaries}

The classical gamma function $\Gamma(v)$ is expressed as follows:

$$
\Gamma(v)=\left\{\begin{array}{lc}
\int_{0}^{\infty} e^{-\mathfrak{u}^{\nu-1} d \mathfrak{u},} & (\Re(v)>0) \\
\frac{\Gamma(v+\kappa)}{(v)_{\kappa}}, & \left(v \in \mathbb{C} \backslash \mathbb{Z}_{0}^{-} ; \kappa \in \mathbb{N}_{0}\right) .
\end{array}\right.
$$

In the above expression $(v)_{\kappa}$ indicates the Pochhammer symbol and is defined (for $v \in \mathbb{C}, \kappa \in \mathbb{N}_{0}$ ) as

$$
(v)_{\kappa}=\frac{\Gamma(v+\kappa)}{\Gamma(v)}=\left\{\begin{array}{lr}
1, & (\kappa=0 ; v \in \mathbb{C} \backslash\{0\}) \\
v(v+1) \cdots(v+n-1), & (\kappa=n \in \mathbb{N} ; v \in \mathbb{C}),
\end{array}\right.
$$

provided that the gamma quotient exists.

The well known IGFs $\gamma(\nu, y)$ and $\Gamma(\nu, y)$ are given in subsequent manner,

$$
\gamma(v, y)=\int_{0}^{y} \mathfrak{u}^{v-1} e^{-\mathfrak{u}} d \mathfrak{u}, \quad(\Re(v)>0 ; y \geq 0)
$$

and

$$
\Gamma(v, y)=\int_{y}^{\infty} \mathfrak{u}^{v-1} e^{-\mathfrak{u}} d \mathfrak{u}, \quad(y \geq 0 ; \Re(v)>0 \text { when } y=0),
$$

respectively and hold the decomposition formula given below:

$$
\gamma(v, y)+\Gamma(v, y)=\Gamma(v), \quad(\Re(v)>0) .
$$

Recently, IHFs $\Gamma_{\mathrm{p}, \mathrm{q}}^{\mathrm{m}, \mathrm{n}}(z)$ and $\gamma_{\mathrm{p}, \mathrm{q}}^{\mathrm{m}, \mathrm{n}}(z)$ were defined by Srivastava et al. ([26] Equations (2.1)-(2.4)) as follows:

$$
\begin{aligned}
& \Gamma_{\mathrm{p}, \mathrm{q}}^{\mathrm{m}, \mathrm{n}}(z)=\Gamma_{\mathrm{p}, \mathrm{q}}^{\mathrm{m}, \mathrm{n}}\left[z \mid \begin{array}{c}
\left(\mathrm{e}_{1}, \mathrm{E}_{1}, y\right),\left(\mathrm{e}_{j}, \mathrm{E}_{j}\right)_{2, \mathrm{p}} \\
\left(\mathrm{f}_{j}, \mathrm{~F}_{j}\right)_{1, \mathrm{q}}
\end{array}\right] \\
& =\Gamma_{p, q}^{m, n}\left[z \mid \begin{array}{c}
\left(e_{1}, E_{1}, y\right),\left(e_{2}, E_{2}\right), \cdots,\left(e_{p}, E_{p}\right) \\
\left(f_{1}, F_{1}\right),\left(f_{2}, F_{2}\right), \cdots,\left(f_{q}, F_{q}\right)
\end{array}\right] \\
& =\frac{1}{2 \pi \mathrm{i}} \int_{\mathfrak{L}} \mathbb{F}(\xi, y) z^{-\xi} d \xi \text {, }
\end{aligned}
$$

where

$$
\mathbb{F}(\xi, y)=\frac{\Gamma\left(1-\mathrm{e}_{1}-\mathrm{E}_{1} \xi, y\right) \prod_{j=1}^{\mathrm{m}} \Gamma\left(\mathrm{f}_{j}+\mathrm{F}_{j} \xi\right) \prod_{j=2}^{\mathrm{n}} \Gamma\left(1-\mathrm{e}_{j}-\mathrm{E}_{j} \xi\right)}{\prod_{j=\mathrm{m}+1}^{\mathrm{q}} \Gamma\left(1-\mathrm{f}_{j}-\mathrm{F}_{j} \xi\right) \prod_{j=\mathrm{n}+1}^{\mathrm{p}} \Gamma\left(\mathrm{e}_{j}+\mathrm{E}_{j} \xi\right)}
$$


and

$$
\begin{aligned}
& \gamma_{\mathrm{p}, \mathrm{q}}^{\mathrm{m}, \mathrm{n}}(z)=\gamma_{\mathrm{p}, \mathrm{q}}^{\mathrm{m}, \mathrm{q}}\left[\begin{array}{c|c}
\left(\mathrm{e}_{1}, \mathrm{E}_{1}, y\right),\left(\mathrm{e}_{j}, \mathrm{E}_{j}\right)_{2, \mathrm{p}} \\
\left(\mathrm{f}_{j}, \mathrm{~F}_{j}\right)_{1, \mathrm{q}}
\end{array}\right]
\end{aligned}
$$

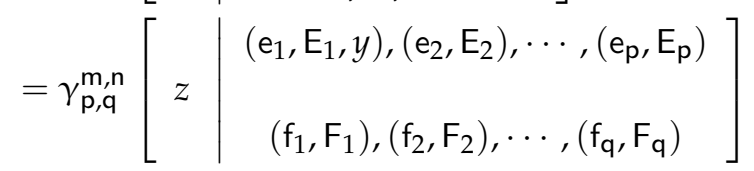

$$
\begin{aligned}
& =\frac{1}{2 \pi \mathrm{i}} \int_{\mathfrak{L}} \mathbb{G}(\xi, y) z^{-\tilde{\zeta}} d \xi \text {, }
\end{aligned}
$$

where

$$
\mathbb{G}(\xi, y)=\frac{\gamma\left(1-\mathrm{e}_{1}-\mathrm{E}_{1} \xi, y\right) \prod_{j=1}^{\mathrm{m}} \Gamma\left(\mathrm{f}_{j}+\mathrm{F}_{j} \xi\right) \prod_{j=2}^{\mathrm{n}} \Gamma\left(1-\mathrm{e}_{j}-\mathrm{E}_{j} \xi\right)}{\prod_{j=\mathrm{m}+1}^{\mathrm{q}} \Gamma\left(1-\mathrm{f}_{j}-\mathrm{F}_{j} \xi\right) \prod_{j=\mathrm{n}+1}^{\mathrm{p}} \Gamma\left(\mathrm{e}_{j}+\mathrm{E}_{j} \xi\right)} .
$$

The IHFs $\Gamma_{\mathrm{p}, \mathrm{q}}^{\mathrm{m}, \mathrm{n}}(z)$ and $\gamma_{\mathrm{p}, \mathrm{q}}^{\mathrm{m}, \mathrm{n}}(z)$ exist for all $y \geq 0$ under the set of conditions as given below:

The contour $\mathfrak{L}$ in the complex $\xi$-plane extends from $\gamma-i \infty$ to $\gamma+i \infty, \gamma \in \mathbb{R}$, and poles of the gamma functions $\Gamma\left(1-\mathrm{e}_{j}-\mathrm{E}_{j} \xi\right), j=\overline{1, \mathrm{n}}$ do not exactly match with the poles of the gamma functions $\Gamma\left(\mathrm{f}_{j}+\mathrm{F}_{j} \xi\right), j=\overline{1, \mathrm{~m}}$. The parameters $\mathrm{m}, \mathrm{n}, \mathrm{p}, \mathrm{q}$ are nonnegative integers satisfying $0 \leq \mathrm{n} \leq \mathrm{p}$, $0 \leq \mathrm{m} \leq \mathrm{q}$. The coefficients $\mathrm{E}_{j}(j=1, \cdots, \mathrm{p})$ and $\mathrm{F}_{j}(j=1, \cdots, q)$ are positive real numbers and $\mathrm{e}_{j}$ and $f_{j}$ are complex. All poles of $\mathbb{F}(\xi, y)$ and $\mathbb{G}(\xi, y)$ are assumed to be simple, and the empty product is taken as unity.

The Mellin-Barnes contour integral in Equation (6) converges absolutely and defines an analytic function for $|\arg (z)|<\frac{1}{2} \mathfrak{H} \pi$, where

$$
\mathfrak{H}=\sum_{j=1}^{\mathrm{n}} \mathrm{E}_{j}+\sum_{j=1}^{\mathrm{m}} \mathrm{F}_{j}-\sum_{j=\mathrm{n}+1}^{\mathrm{p}} \mathrm{E}_{j}-\sum_{j=\mathrm{m}+1}^{\mathrm{q}} \mathrm{F}_{j}>0 .
$$

Remark 1. Numerous special functions can be obtained by specializing the parameters of IHFs; some of them can be found in the articles given by Bansal and Choi [22] and Bansal et al. [23].

The incomplete $\bar{H}$-functions $\bar{\gamma}_{p, q}^{m, n}(z)$ and $\bar{\Gamma}_{p, q}^{m, n}(z)$ were defined by Srivastava et al. ([26] p. 134, Equations (7.5)-(7.8)) as follows:

$$
\begin{aligned}
& \bar{\Gamma}_{p, q}^{m, n}(z)=\bar{\Gamma}_{p, q}^{m, n}[z\left.\begin{array}{c}
\left(g_{1}, G_{1} ; \epsilon_{1}: y\right),\left(g_{j}, G_{j} ; \epsilon_{j}\right)_{2, n},\left(g_{j}, G_{j}\right)_{n+1, p} \\
\left(h_{j}, H_{j}\right)_{1, m},\left(h_{j}, H_{j} ; \mathcal{K}_{j}\right)_{m+1, q}
\end{array}\right] \\
&:=\frac{1}{2 \pi i} \int_{\mathfrak{L}} \bar{f}(\xi, y) z^{-\xi} d \xi,
\end{aligned}
$$

where

$$
\bar{f}(\xi, y)=\frac{\left[\Gamma\left(1-g_{1}-G_{1} \xi, y\right)\right]^{\epsilon_{1}} \prod_{j=1}^{m} \Gamma\left(h_{j}+H_{j} \xi\right) \prod_{j=2}^{n}\left[\Gamma\left(1-g_{j}-G_{j} \xi\right)\right]_{j}^{\epsilon_{j}}}{\prod_{j=m+1}^{q}\left[\Gamma\left(1-h_{j}-H_{j} \xi\right)\right]^{\mathcal{K}_{j}} \prod_{j=n+1}^{p} \Gamma\left(g_{j}+G_{j} \xi\right)}
$$


and

$$
\begin{aligned}
\bar{\gamma}_{p, q}^{m, n}(z)= & \bar{\gamma}_{p, q}^{m, n}\left[z \mid \begin{array}{c}
\left(g_{1}, G_{1} ; \in_{1}: y\right),\left(g_{j}, G_{j} ; \in_{j}\right)_{2, n},\left(g_{j}, G_{j}\right)_{n+1, p} \\
\left(h_{j}, H_{j}\right)_{1, m},\left(h_{j}, H_{j} ; \mathcal{K}_{j}\right)_{m+1, q}
\end{array}\right] \\
= & \frac{1}{2 \pi i} \int_{\mathfrak{L}} \bar{F}(\xi, y) z^{-\xi} d \xi
\end{aligned}
$$

where

$$
\bar{F}(\xi, y)=\frac{\left[\gamma\left(1-g_{1}-G_{1} \xi, y\right)\right]^{\epsilon_{1}} \prod_{j=1}^{m} \Gamma\left(h_{j}+H_{j} \xi\right) \prod_{j=2}^{n}\left[\Gamma\left(1-g_{j}-G_{j} \xi\right)\right]^{\epsilon_{j}}}{\prod_{j=m+1}^{q}\left[\Gamma\left(1-h_{j}-H_{j} \xi\right)\right]^{\mathcal{K}_{j}} \prod_{j=n+1}^{p} \Gamma\left(g_{j}+G_{j} \xi\right)} .
$$

The conditions of incomplete $\bar{H}$-functions $\bar{\gamma}_{p, q}^{m, n}(z)$ and $\bar{\Gamma}_{p, q}^{m, n}(z)$ are stated in the articles given by Srivasatava et al. [26] and Inayat-Hussain [30].

Remark 2. A variety of special functions can be obtained by specializing the parameters of incomplete $\bar{H}$-functions; some of them can be found in the articles given by Kumar et al. [27].

Next, we give two important result, which are used in our main results.

1. The Mellin transform of IHF ([26] p.122, Equation (3.4)) is defined as follows:

$$
\mathfrak{M}\left\{\Gamma_{\mathrm{p}, \mathrm{q}}^{\mathrm{m}, \mathrm{q}}\left[\begin{array}{c|c}
z t^{\sigma} & \left.\begin{array}{c}
\left(\mathrm{e}_{1}, \mathrm{E}_{1}, y\right),\left(\mathrm{e}_{j}, \mathrm{E}_{j}\right)_{2, \mathrm{p}} \\
\left(\mathrm{f}_{j}, \mathrm{~F}_{j}\right)_{1, \mathrm{q}}
\end{array}\right] ; \xi
\end{array}\right\}=\frac{z^{-\frac{\xi}{\sigma}}}{\sigma} \mathbb{F}\left(\frac{\xi}{\sigma}, y\right)\right.
$$

where $\mathbb{F}\left(\frac{\xi}{\sigma}, y\right)$ is given in Equation (7) and the conditions are given in [26].

2. The Mellin transform of incomplete $\bar{H}$-function ([26] p.122, Equation (3.4)) is defined as follows:

$$
\mathfrak{M}\left\{\bar{\Gamma}_{p, q}^{m, n}\left[z t^{\mu} \mid \begin{array}{c}
\left(g_{1}, G_{1} ; \epsilon_{1}: y\right),\left(g_{j}, G_{j} ; \epsilon_{j}\right)_{2, n},\left(g_{j}, G_{j}\right)_{n+1, p} \\
\left(h_{j}, H_{j}\right)_{1, m},\left(h_{j}, H_{j} ; \mathcal{K}_{j}\right)_{m+1, q}
\end{array}\right] ; \xi\right\}=\frac{z^{-\frac{\xi}{\mu}}}{\mu} \bar{f}\left(\frac{\xi}{\mu}, y\right),
$$

where $\bar{f}\left(\frac{\xi}{\mu}, y\right)$ is given in Equation (12) and the conditions are given in [26].

Suppose $\mathfrak{A}$ denotes the space of all functions $f$ which are well defined on $\mathbb{R}=[0, \infty)$ and satisfy

(i) $f \in \wp(\mathbb{R})$;

(ii) $\lim _{z \rightarrow \infty}\left\{z^{\alpha} f^{k}(z)\right\}=0, \quad(\forall \alpha, \mathrm{k}$ is a nonnegative integers $)$; and

(iii) $f(z)=O(1), \quad z \rightarrow 0$.

For correspondence to the space of good functions defined on the whole real line $(-\infty, \infty)$, see the work of Lighthill [31].

The Weyl fractional integral operator of order $\alpha$ [32] is expressed as

$$
W^{-\alpha}\{f(z)\}=\frac{1}{\Gamma(\alpha)} \int_{z}^{\infty}(\tau-z)^{\alpha-1} f(\tau) d \tau, \quad(\Re(\alpha)>0, f \in \mathfrak{A}) .
$$

It should also be noted that, in all the lemmas and theorems, the factors $t^{-k}$ and $t^{-\lambda}$ are absorbed in $f$, but they are there to keep the formulas as graceful as possible. 


\section{Solution of Fredholm-Type Integral Equation Involving Incomplete H-Functions}

In this part, we give the solution of Fredholm-type integral equation involving the Incomplete H-functions with the help of the Mellin transform technique and the well known Weyl fractional operator.

Lemma 1. Let

(i) $\mathrm{M}, \mathrm{N}, \mathrm{P}, \mathrm{Q}$ are nonnegative integers such that (s.t.) $1 \leq \mathrm{M} \leq \mathrm{Q}$ and $0 \leq \mathrm{N} \leq \mathrm{P}$.

(ii) $\Re(\lambda-k)>0 ; \Re\left[k+\sigma\left(\frac{f_{j}}{F_{j}}\right)\right]>0 \quad(j=1, \cdots, M)$.

(iii) $y \geq 0, \sigma>0$ and $\lambda \in \mathbb{C}$.

(iv) $|\arg (A)|<\frac{1}{2} \mathfrak{H} \pi$, where $\mathfrak{H}$ is given by Equation (10).

Then,



$$
\begin{aligned}
& =t^{-k} \Gamma_{\mathrm{P}+1, \mathrm{Q}+1}^{\mathrm{M}, \mathrm{N}+1}\left[A\left(\frac{x}{t}\right)^{\sigma} \mid \begin{array}{c}
\left(\mathrm{e}_{1}, \mathrm{E}_{1}, y\right),(1-k, \sigma),\left(\mathrm{e}_{j}, \mathrm{E}_{j}\right)_{2, \mathrm{P}} \\
\left(\mathrm{f}_{j}, \mathrm{~F}_{j}\right)_{1, \mathrm{Q}},(1-\lambda, \sigma)
\end{array}\right] \text {. }
\end{aligned}
$$

Proof. To prove the assertion in Equation (18), we first employ the Weyl fractional integral presented in Equation (17), express the contour integral form of the IHF given in Equation (6), and then change the order of integrals (under the stated permissible conditions ). Next, evaluating the Weyl fractional integral of power function and then reinterpreting the resulting Mellin-Barnes contour integral in terms of IHF, we easily achieve the required result.

\section{Lemma 2. Let}

(i) $\mathrm{M}, \mathrm{N}, \mathrm{P}, \mathrm{Q}$ are nonnegative integers s.t. $1 \leq \mathrm{M} \leq \mathrm{Q}$ and $0 \leq \mathrm{N} \leq \mathrm{P}$.

(ii) $\Re(\lambda-k)>0 ; \Re\left[k+\sigma\left(\frac{f_{j}}{F_{j}}\right)\right]>0 \quad(j=1, \cdots, M)$.

(iii) $y \geq 0, \sigma>0$ and $\lambda \in \mathbb{C}$.

(iv) $|\arg (A)|<\frac{1}{2} \mathfrak{H} \pi$, where $\mathfrak{H}$ is given by Equation (10).

Then,



$$
\begin{aligned}
& =t^{-k} \gamma_{\mathrm{P}+1, \mathrm{Q}+1}^{\mathrm{M}+\mathrm{N}+1}\left[A\left(\frac{x}{t}\right)^{\sigma} \mid \begin{array}{c}
\left(\mathrm{e}_{1}, \mathrm{E}_{1}, y\right),(1-k, \sigma),\left(\mathrm{e}_{j}, \mathrm{E}_{j}\right)_{2, \mathrm{P}} \\
\left(\mathrm{f}_{j}, \mathrm{~F}_{j}\right)_{1, \mathrm{Q}},(1-\lambda, \sigma)
\end{array}\right] \text {. }
\end{aligned}
$$

Proof. To prove the assertion in Equation (19), we first utilize the Weyl fractional integral written in Equation (17), express the contour integral form of the IHF given in Equation (8), and then change the order of integrals (under the stated permissible conditions ). Next, evaluating the Weyl fractional integral of power function and then reinterpreting the resulting Mellin-Barnes contour integral in terms of IHF, we easily achieve the required result.

Theorem 1. Let

(i) $\mathrm{M}, \mathrm{N}, \mathrm{P}, \mathrm{Q}$ are nonnegative integers s.t. $1 \leq \mathrm{M} \leq \mathrm{Q}$ and $0 \leq \mathrm{N} \leq \mathrm{P}$. 
(ii) $\Re\left(k+\sigma\left(\frac{\mathrm{e}_{\mathrm{j}}-1}{\mathrm{E}_{\mathrm{j}}}\right)\right)<0 \quad(j=1, \cdots, N) ; \Re\left[k+\sigma\left(\frac{\mathrm{f}_{\mathrm{j}}}{\mathrm{F}_{\mathrm{j}}}\right)\right]>0 \quad(j=1, \cdots, M)$.

(iii) $y \geq 0, \sigma>0$, and $\lambda \in \mathbb{C}$.

Then, the following integral relation formula holds:

$$
\left.\begin{array}{c}
\int_{0}^{\infty} t^{-k} \Gamma_{\mathrm{P}+1, \mathrm{Q}+1}^{\mathrm{M}, \mathrm{N}+1}\left[A\left(\frac{x}{t}\right)^{\sigma}\right. \\
\quad=\int_{0}^{\infty} t^{-\lambda} \Gamma_{\mathrm{P}, \mathrm{Q}}^{\mathrm{M}, \mathrm{N}}\left[A\left(\frac{x}{t}\right)^{\sigma}\right. \\
\left(\mathrm{f}_{j}, \mathrm{~F}_{j}\right)_{1, \mathrm{Q}},(1-\lambda, \sigma)
\end{array}\right] f(t) d t
$$

provided that $f \in \mathfrak{A}$ and $x>0$.

Proof. Let $\Omega$ indicate the first member of the assertion in Equation (20) of Theorem 1. Then, by Lemma 1 and the definition in Equation (17), we have



Next, changing the order of integration under the permissible conditions, we get

$$
\Omega=\int_{0}^{\infty} u^{-\lambda} \Gamma_{\mathrm{P}, \mathrm{Q}}^{\mathrm{M}, \mathrm{N}}\left[A\left(\frac{x}{u}\right)^{\sigma} \mid \begin{array}{c}
\left(\mathrm{e}_{1}, \mathrm{E}_{1}, y\right),\left(\mathrm{e}_{j}, \mathrm{E}_{j}\right)_{2, \mathrm{P}} \\
\left(\mathrm{f}_{j}, \mathrm{~F}_{j}\right)_{1, \mathrm{Q}}
\end{array}\right]\left(\int_{0}^{u} \frac{(u-t)^{\lambda-k-1}}{\Gamma(\lambda-k)} f(t) d t\right) d u .
$$

Further, with the help of well known definition of Riemann-Liouville (RL) fractional derivative operator, we obtain



which is the right-hand member of Equation (20).

\section{Theorem 2. Let}

(i) $\mathrm{M}, \mathrm{N}, \mathrm{P}, \mathrm{Q}$ are nonnegative integers s.t. $1 \leq \mathrm{M} \leq \mathrm{Q}$ and $0 \leq \mathrm{N} \leq \mathrm{P}$.

(ii) $\Re\left(k+\sigma\left(\frac{\mathrm{e}_{\mathrm{j}}-1}{\mathrm{E}_{\mathrm{j}}}\right)\right)<0 \quad(j=1, \cdots, N) ; \Re\left[k+\sigma\left(\frac{\mathrm{f}_{\mathrm{j}}}{\mathrm{F}_{\mathrm{j}}}\right)\right]>0 \quad(j=1, \cdots, M)$.

(iii) $y \geq 0, \sigma>0$, and $\lambda \in \mathbb{C}$.

Then, the following integral relation formula holds:

$$
\left.\begin{array}{c}
\int_{0}^{\infty} t^{-k} \gamma_{\mathrm{P}+1, \mathrm{Q}+1}^{\mathrm{M}, \mathrm{N}+1}\left[A\left(\frac{x}{t}\right)^{\sigma}\right. \\
=\int_{0}^{\infty} t^{-\lambda} \gamma_{\mathrm{P}, \mathrm{Q}}^{\mathrm{M}, \mathrm{N}}\left[A\left(\frac{x}{t}\right)^{\sigma}\right. \\
\left(\mathrm{f}_{j}, \mathrm{~F}_{j}\right)_{1, \mathrm{Q}},(1-\lambda, \sigma)
\end{array}\right] f(t) d t
$$

provided that $f \in \mathfrak{A}$ and $x>0$. 
Proof. We here omit the details since the proof is parallel to that of Theorem 1.

Theorem 3. Let

(i) $\mathrm{M}, \mathrm{N}, \mathrm{P}, \mathrm{Q}$ are nonnegative integers s.t. $1 \leq \mathrm{M} \leq \mathrm{Q}$ and $0 \leq \mathrm{N} \leq \mathrm{P}$.

(ii) $\Re\left(\lambda+\sigma\left(\frac{e_{j}-1}{E_{j}}\right)\right)<0 \quad(j=1, \cdots, N) ; \Re\left[\lambda+\sigma\left(\frac{f_{j}}{F_{j}}\right)\right]>0 \quad(j=1, \cdots, M)$.

(iii) $-\min _{1 \leq j \leq M}\left(\frac{\Re\left(\mathrm{f}_{j}\right)}{\mathrm{F}_{j}}\right)<\Re(\xi)<\min _{1 \leq j \leq N}\left(\frac{\Re\left(1-\mathrm{e}_{j}\right)}{\mathrm{E}_{j}}\right)$.

(iv) $y \geq 0, \sigma>0, f, g \in \mathfrak{A}$ and $\lambda \in \mathbb{C}$.

Then, the following integral equation

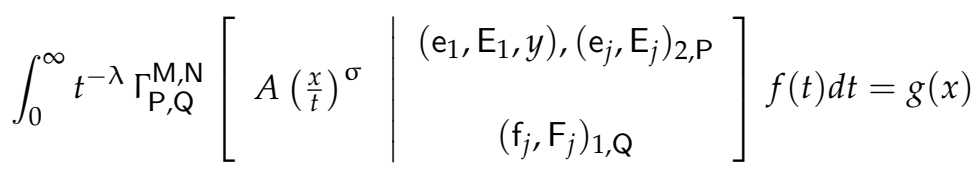

has a solution given by

$$
f(x)=\frac{\sigma x^{\lambda-1}}{2 \pi i} \int_{\mathcal{L}}\left[\mathbb{F}\left(\frac{\xi}{\sigma}, y\right)\right]^{-1} x^{-\xi} \varphi(\xi) d \xi
$$

where

$$
\varphi(\xi)=\int_{0}^{\infty} x^{\xi-1} g(x) d x
$$

and $\mathbb{F}(\xi, y)$ is given in Equation (7).

Proof. To find the solution of integral equation in Equation (22), $\mathrm{f}$ is replaced by $D^{\lambda-k} f$ in Equation (20); we have

$$
\int_{0}^{\infty} t^{-k} \Gamma_{\mathrm{P}+1, \mathrm{Q}+1}^{\mathrm{M}, \mathrm{N}+1}\left[A\left(\frac{x}{t}\right)^{\sigma} \mid \begin{array}{c}
\left(\mathrm{e}_{1}, \mathrm{E}_{1}, y\right),(1-k, \sigma),\left(\mathrm{e}_{j}, \mathrm{E}_{j}\right)_{2, \mathrm{P}} \\
\left(\mathrm{f}_{j}, \mathrm{~F}_{j}\right)_{1, \mathrm{Q}},(1-\lambda, \sigma)
\end{array}\right] D^{\lambda-k}\{f(t)\} d t=g(x) .
$$

Multiplying both sides by $x^{\xi-1}$ and integrating with respect to $\mathrm{x}$ from 0 to $\infty$ then changing the order of integration under the permissible conditions, we have

$$
\begin{aligned}
& \varphi(\xi)=\int_{0}^{\infty} x^{\xi-1} g(x) d x=\int_{0}^{\infty} t^{-k} D^{\lambda-k}\{f(t)\}
\end{aligned}
$$

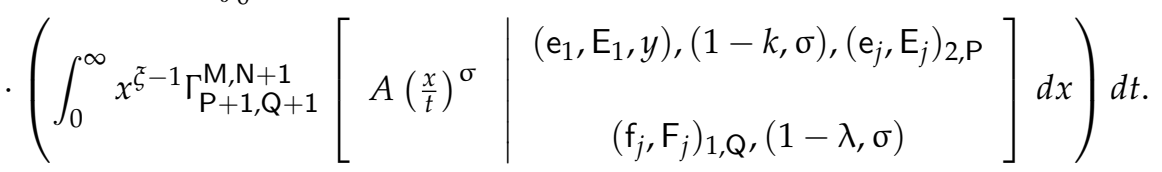

Now, with the help of Equation (15), we get

$$
\varphi(\xi)=\frac{\Gamma(k-\xi)}{\sigma \Gamma(\lambda-\xi)} A^{-\frac{\xi}{\sigma}} \mathbb{F}\left(\frac{\xi}{\sigma}, y\right) \int_{0}^{\infty} t^{\xi-k} D^{\lambda-k}\{f(t)\} d t
$$

Further, applying the well known Mellin inversion theorem, we get

$$
D^{\lambda-k}\{f(t)\}=\frac{\sigma}{2 \pi i} \int_{\mathcal{L}} t^{k-\xi-1} \frac{\Gamma(\lambda-\xi)}{\Gamma(k-\xi)} A^{\frac{\xi}{\sigma}}\left[\mathbb{F}\left(\frac{\xi}{\sigma}, y\right)\right]^{-1} \varphi(\xi) d \xi
$$


Next, operating both sides by $D^{k-\lambda}$, we have

$$
f(t)=\frac{\sigma}{2 \pi i} D^{k-\lambda}\left\{\int_{\mathcal{L}} t^{k-\xi-1} \frac{\Gamma(\lambda-\xi)}{\Gamma(k-\xi)} A^{\frac{\xi}{\sigma}}\left[\mathbb{F}\left(\frac{\xi}{\sigma}, y\right)\right]^{-1} \varphi(\xi) d \xi\right\},
$$

which finally yields

$$
f(x)=\frac{\sigma x^{\lambda-1}}{2 \pi i} \int_{\mathcal{L}}\left[\mathbb{F}\left(\frac{\xi}{\sigma}, y\right)\right]^{-1} x^{-\xi} \varphi(\xi) d \xi
$$

Theorem 4. Let

(i) $\mathrm{M}, \mathrm{N}, \mathrm{P}, \mathrm{Q}$ are nonnegative integers s.t. $1 \leq \mathrm{M} \leq \mathrm{Q}$ and $0 \leq \mathrm{N} \leq \mathrm{P}$.

(ii) $\Re\left(\lambda+\sigma\left(\frac{\mathrm{e}_{\mathrm{j}}-1}{\mathrm{E}_{\mathrm{j}}}\right)\right)<0 \quad(j=1, \cdots, N) ; \Re\left[\lambda+\sigma\left(\frac{\mathrm{f}_{\mathrm{j}}}{\mathrm{F}_{\mathrm{j}}}\right)\right]>0 \quad(j=1, \cdots, M)$.

(iii) $-\min _{1 \leq j \leq M}\left(\frac{\Re\left(\mathrm{f}_{j}\right)}{\mathrm{F}_{j}}\right)<\Re(\xi)<\min _{1 \leq j \leq N}\left(\frac{\Re\left(1-\mathrm{e}_{j}\right)}{\mathrm{E}_{j}}\right)$.

(iv) $y \geq 0, \sigma>0, f, g \in \mathfrak{A}$ and $\lambda \in \mathbb{C}$.

Then, the following integral equation

$$
\int_{0}^{\infty} t^{-\lambda} \gamma_{\mathrm{P}, \mathrm{Q}}^{\mathrm{M}}\left[A\left(\frac{x}{t}\right)^{\sigma} \mid \begin{array}{c}
\left(\mathrm{e}_{1}, \mathrm{E}_{1}, y\right),\left(\mathrm{e}_{j}, \mathrm{E}_{j}\right)_{2, \mathrm{P}} \\
\left(\mathrm{f}_{j}, \mathrm{~F}_{j}\right)_{1, \mathrm{Q}}
\end{array}\right] f(t) d t=g(x)
$$

has a solution given by

$$
f(x)=\frac{\sigma x^{\lambda-1}}{2 \pi i} \int_{\mathcal{L}}\left[\mathbb{G}\left(\frac{\xi}{\sigma}, y\right)\right]^{-1} x^{-\xi} \varphi(\xi) d \xi,
$$

where

$$
\varphi(\xi)=\int_{0}^{\infty} x^{\xi-1} g(x) d x
$$

and $\mathbb{G}(\xi, y)$ is given in Equation (9).

Proof. We here omit the details since the proof is parallel to that of Theorem 3.

\section{Solution of Fredholm-Type Integral Equation Involving Incomplete $\bar{H}$-Function}

In this portion, we give the solution of Fredholm-type integral equation involving the Incomplete $\bar{H}$-functions with the help of Mellin transform technique and well known Weyl fractional operator.

Lemma 3. Let

(i) $m, n, p, q$ are nonnegative integers s.t. $1 \leq m \leq q$ and $0 \leq n \leq p$.

(ii) $\Re(\lambda-k)>0 ; \Re\left[k+\sigma\left(\frac{h_{j}}{H_{j}}\right)\right]>0 \quad(j=1, \cdots, m)$.

(iii) $y \geq 0, \sigma>0$ and $\lambda \in \mathbb{C}$. 
Then,

$$
\begin{aligned}
& W^{k-\lambda}\left\{t^{-\lambda} \bar{\Gamma}_{p, q}^{m, n}\left[A\left(\frac{x}{t}\right)^{\sigma} \mid \begin{array}{c}
\left(g_{1}, G_{1} ; \epsilon_{1}: y\right),\left(g_{j}, G_{j} ; \epsilon_{j}\right)_{2, n},\left(g_{j}, G_{j}\right)_{n+1, p} \\
\left(h_{j}, H_{j}\right)_{1, m},\left(h_{j}, H_{j} ; \mathcal{K}_{j}\right)_{m+1, q}
\end{array}\right]\right\} \\
& =t^{-k} \bar{\Gamma}_{p+1, q+1}^{m, n+1}\left[A\left(\frac{x}{t}\right)^{\sigma} \mid \begin{array}{c}
\left(g_{1}, G_{1} ; \in_{1}: y\right),(1-k, \sigma ; 1),\left(g_{j}, G_{j} ; \epsilon_{j}\right)_{2, n},\left(g_{j}, G_{j}\right)_{n+1, p} \\
\left(h_{j}, H_{j}\right)_{1, m},(1-\lambda, \sigma ; 1),\left(h_{j}, H_{j} ; \mathcal{K}_{j}\right)_{m+1, q}
\end{array}\right] .
\end{aligned}
$$

Proof. To prove the assertion in Equation (26), we first employ the Weyl fractional integral written in Equation (17), express the contour integral form of the incomplete $\bar{H}$-function given in Equation (11), and then change the order of integrals (under the stated permissible conditions ). Next, evaluating the Weyl fractional integral of power function and then reinterpreting the resulting Mellin-Barnes contour integral in terms of incomplete $\bar{H}$-function, we easily achieve the required result.

\section{Lemma 4. Let}

(i) $m, n, p, q$ are nonnegative integers s.t. $1 \leq m \leq q$ and $0 \leq n \leq p$.

(ii) $\Re(\lambda-k)>0 ; \Re\left[k+\sigma\left(\frac{h_{j}}{H_{j}}\right)\right]>0 \quad(j=1, \cdots, m)$.

(iii) $y \geq 0, \sigma>0$ and $\lambda \in \mathbb{C}$.

Then,

$$
\begin{gathered}
W^{k-\lambda}\left\{t^{-\lambda} \bar{\gamma}_{p, q}^{m, n}\left[A\left(\frac{x}{t}\right)^{\sigma} \mid \begin{array}{c}
\left(g_{1}, G_{1} ; \in_{1}: y\right),\left(g_{j}, G_{j} ; \epsilon_{j}\right)_{2, n},\left(g_{j}, G_{j}\right)_{n+1, p} \\
\left(h_{j}, H_{j}\right)_{1, m},\left(h_{j}, H_{j} ; \mathcal{K}_{j}\right)_{m+1, q}
\end{array}\right]\right\} \\
=t^{-k} \bar{\gamma}_{p+1, q+1}^{m, n+1}\left[A\left(\frac{x}{t}\right)^{\sigma} \mid \begin{array}{c}
\left(g_{1}, G_{1} ; \in_{1}: y\right),(1-k, \sigma ; 1),\left(g_{j}, G_{j} ; \in_{j}\right)_{2, n},\left(g_{j}, G_{j}\right)_{n+1, p} \\
\left(h_{j}, H_{j}\right)_{1, m},(1-\lambda, \sigma ; 1),\left(h_{j}, H_{j} ; \mathcal{K}_{j}\right)_{m+1, q}
\end{array}\right] .
\end{gathered}
$$

Proof. To prove the assertion in Equation (26), we first employ the definition of Weyl fractional integral expressed in Equation (17), express the contour integral form of the incomplete $\bar{H}$-function given in Equation (13), and then change the order of integrals (under the stated permissible conditions). Next, evaluating the Weyl fractional integral of power function and then reinterpreting the resulting Mellin-Barnes contour integral in terms of incomplete $\bar{H}$-function, we easily achieve the needed result.

\section{Theorem 5. Let}

(i) $m, n, p, q$ are nonnegative integers s.t. $1 \leq m \leq q$ and $0 \leq n \leq p$.

(ii) $\Re\left(k+\sigma\left(\frac{\mathrm{g}_{\mathrm{j}}-1}{\mathrm{G}_{\mathrm{j}}}\right)\right)<0 \quad(j=1, \cdots, n) ; \Re\left[k+\sigma\left(\frac{\mathrm{h}_{\mathrm{j}}}{\mathrm{H}_{\mathrm{j}}}\right)\right]>0 \quad(j=1, \cdots, m)$.

(iii) $y \geq 0, \sigma>0$ and $\lambda \in C$.

Then, the following integral relation formula holds:

$$
\begin{aligned}
& \int_{0}^{\infty} t^{-k} \bar{\Gamma}_{p+1, q+1}^{m, n+1}\left[A\left(\frac{x}{t}\right)^{\sigma} \mid \begin{array}{c}
\left(g_{1}, G_{1} ; \epsilon_{1}: y\right),(1-k, \sigma ; 1),\left(g_{j}, G_{j} ; \epsilon_{j}\right)_{2, n},\left(g_{j}, G_{j}\right)_{n+1, p} \\
\left(h_{j}, H_{j}\right)_{1, m},(1-\lambda, \sigma ; 1),\left(h_{j}, H_{j} ; \mathcal{K}_{j}\right)_{m+1, q}
\end{array}\right] f(t) d t \\
& =\int_{0}^{\infty} t^{-\lambda} \bar{\Gamma}_{p, q}^{m, n}\left[A\left(\frac{x}{t}\right)^{\sigma} \mid \begin{array}{c}
\left(g_{1}, G_{1} ; \epsilon_{1}: y\right),\left(g_{j}, G_{j} ; \epsilon_{j}\right)_{2, n},\left(g_{j}, G_{j}\right)_{n+1, p} \\
\left(h_{j}, H_{j}\right)_{1, m},\left(h_{j}, H_{j} ; \mathcal{K}_{j}\right)_{m+1, q}
\end{array}\right] D^{k-\lambda}\{f(t)\} d t
\end{aligned}
$$


provided $f \in \mathfrak{A}$ and $x>0$.

Proof. Let $\bar{\Omega}$ indicate the first member of the assertion in Equation (28) of Theorem 5 . Then, by Lemma 3 and the definition in Equation (17), we have

$$
\begin{aligned}
& \Omega=\int_{0}^{\infty} f(t) \int_{t}^{\infty} \frac{(u-t)^{\lambda-k-1}}{\Gamma(\lambda-k)} u^{-\lambda} \\
& \times \bar{\Gamma}_{p, q}^{m, n}\left[A\left(\frac{x}{u}\right)^{\sigma} \mid \begin{array}{c}
\left(g_{1}, G_{1} ; \in_{1}: y\right),\left(g_{j}, G_{j} ; \epsilon_{j}\right)_{2, n},\left(g_{j}, G_{j}\right)_{n+1, p} \\
\left(h_{j}, H_{j}\right)_{1, m},\left(h_{j}, H_{j} ; \mathcal{K}_{j}\right)_{m+1, q}
\end{array}\right] d u d t .
\end{aligned}
$$

Next, changing the order of integration under the permissible conditions, we achieve the result below:

$$
\begin{aligned}
\Omega=\int_{0}^{\infty} u^{-\lambda} \bar{\Gamma}_{p, q}^{m, n} & {\left[A\left(\frac{x}{u}\right)^{\sigma} \mid \begin{array}{r}
\left(g_{1}, G_{1} ; \in_{1}: y\right),\left(g_{j}, G_{j} ; \epsilon_{j}\right)_{2, n},\left(g_{j}, G_{j}\right)_{n+1, p} \\
\left(h_{j}, H_{j}\right)_{1, m},\left(h_{j}, H_{j} ; \mathcal{K}_{j}\right)_{m+1, q}
\end{array}\right] } \\
& \times\left(\int_{0}^{u} \frac{(u-t)^{\lambda-k-1}}{\Gamma(\lambda-k)} f(t) d t\right) d u .
\end{aligned}
$$

Further, with the help of well known definition of RL fractional derivative operator, we obtain

$$
\Omega=\int_{0}^{\infty} u^{-\lambda} \bar{\Gamma}_{p, q}^{m, n}\left[A\left(\frac{x}{u}\right)^{\sigma} \mid \begin{array}{c}
\left(g_{1}, G_{1} ; \in_{1}: y\right),\left(g_{j}, G_{j} ; \in_{j}\right)_{2, n},\left(g_{j}, G_{j}\right)_{n+1, p} \\
\left(h_{j}, H_{j}\right)_{1, m},\left(h_{j}, H_{j} ; \mathcal{K}_{j}\right)_{m+1, q}
\end{array}\right] D^{k-\lambda}\{f(u)\} d u
$$

which is the right-hand member of Equation (20).

Theorem 6. Let

(i) $m, n, p, q$ are nonnegative integers s.t. $1 \leq m \leq q$ and $0 \leq n \leq p$.

(ii) $\Re\left(k+\sigma\left(\frac{\mathrm{g}_{\mathrm{j}}-1}{\mathrm{G}_{\mathrm{j}}}\right)\right)<0 \quad(j=1, \cdots, n) ; \Re\left[k+\sigma\left(\frac{\mathrm{h}_{\mathrm{j}}}{\mathrm{H}_{\mathrm{j}}}\right)\right]>0 \quad(j=1, \cdots, m)$.

(iii) $y \geq 0, \sigma>0$ and $\lambda \in \mathbb{C}$.

Then, the following integral relation formula holds:

$$
\begin{aligned}
& \int_{0}^{\infty} t^{-k} \bar{\gamma}_{p+1, q+1}^{m, n+1}\left[A\left(\frac{x}{t}\right)^{\sigma} \mid \begin{array}{c}
\left(g_{1}, G_{1} ; \epsilon_{1}: y\right),(1-k, \sigma ; 1),\left(g_{j}, G_{j} ; \epsilon_{j}\right)_{2, n},\left(g_{j}, G_{j}\right)_{n+1, p} \\
\left(h_{j}, H_{j}\right)_{1, m},(1-\lambda, \sigma ; 1),\left(h_{j}, H_{j} ; \mathcal{K}_{j}\right)_{m+1, q}
\end{array}\right] f(t) d t \\
& =\int_{0}^{\infty} t^{-\lambda} \bar{\gamma}_{p, q}^{m, n}\left[A\left(\frac{x}{t}\right)^{\sigma} \mid \begin{array}{c}
\left(g_{1}, G_{1} ; \epsilon_{1}: y\right),\left(g_{j}, G_{j} ; \epsilon_{j}\right)_{2, n},\left(g_{j}, G_{j}\right)_{n+1, p} \\
\left(h_{j}, H_{j}\right)_{1, m},\left(h_{j}, H_{j} ; \mathcal{K}_{j}\right)_{m+1, q}
\end{array}\right] D^{k-\lambda}\{f(t)\} d t .
\end{aligned}
$$

provided $f \in \mathfrak{A}$ and $x>0$.

Proof. We here omit the details since the proof is parallel to that of Theorem 5.

\section{Theorem 7. Let}

(i) $m, n, p, q$ are nonnegative integers s.t. $1 \leq m \leq q$ and $0 \leq n \leq p$.

(ii) $\Re\left(k+\sigma\left(\frac{\mathrm{g}_{\mathrm{j}}-1}{\mathrm{G}_{\mathrm{j}}}\right)\right)<0 \quad(j=1, \cdots, n) ; \Re\left[k+\sigma\left(\frac{\mathrm{h}_{\mathrm{j}}}{\mathrm{H}_{\mathrm{j}}}\right)\right]>0 \quad(j=1, \cdots, m)$. 
(iii) $-\min _{1 \leq j \leq m}\left(\frac{\Re\left(h_{j}\right)}{H_{j}}\right)<\Re(\xi)<\min _{1 \leq j \leq n}\left(\frac{\Re\left(1-g_{j}\right)}{G_{j}}\right)$.

(iv) $y \geq 0, \sigma>0, \lambda \in \mathbb{C}$ and $f, g \in \mathfrak{A}$.

Then, the following integral equation

$$
\int_{0}^{\infty} t^{-\lambda} \bar{\Gamma}_{p, q}^{m, n}\left[A\left(\frac{x}{t}\right)^{\sigma} \mid \begin{array}{c}
\left(g_{1}, G_{1} ; \epsilon_{1}: y\right),\left(g_{j}, G_{j} ; \in_{j}\right)_{2, n},\left(g_{j}, G_{j}\right)_{n+1, p} \\
\left(h_{j}, H_{j}\right)_{1, m},\left(h_{j}, H_{j} ; \mathcal{K}_{j}\right)_{m+1, q}
\end{array}\right] f(t) d t=g(x)
$$

has a solution given by

$$
f(x)=\frac{\sigma x^{\lambda-1}}{2 \pi i} \int_{\mathcal{L}}\left[\bar{f}\left(\frac{\xi}{\sigma}, y\right)\right]^{-1} x^{-\xi} \varphi(\xi) d \xi
$$

where

$$
\varphi(\xi)=\int_{0}^{\infty} x^{\xi-1} g(x) d x
$$

and $\bar{f}(\xi, y)$ is given in Equation (12).

Proof. To determine the solution of the integral equation in Equation (30), $\mathrm{f}$ is replaced by $D^{\lambda-k} f$ in Equation (28); we have

$$
\begin{aligned}
& \int_{0}^{\infty} t^{-k} \bar{\Gamma}_{p+1, q+1}^{m, n+1}\left[A\left(\frac{x}{t}\right)^{\sigma}\right.\left.\begin{array}{c}
\left(g_{1}, G_{1} ; \epsilon_{1}: y\right),(1-k, \sigma ; 1),\left(g_{j}, G_{j} ; \epsilon_{j}\right)_{2, n},\left(g_{j}, G_{j}\right)_{n+1, p} \\
\left(h_{j}, H_{j}\right)_{1, m},(1-\lambda, \sigma ; 1),\left(h_{j}, H_{j} ; \mathcal{K}_{j}\right)_{m+1, q}
\end{array}\right] \\
& \times D^{\lambda-k}\{f(t)\} d t=g(x) .
\end{aligned}
$$

Multiplying both sides by $x^{\xi-1}$ and integrating with respect to $\mathrm{x}$ from 0 to $\infty$ then changing the order of integration under the permissible conditions, we have

$$
\begin{aligned}
\varphi(\xi) & =\int_{0}^{\infty} x^{\xi-1} g(x) d x=\int_{0}^{\infty} t^{-k} D^{\lambda-k}\{f(t)\} \\
& \cdot\left(\int _ { 0 } ^ { \infty } x ^ { \xi - 1 } \overline { \Gamma } _ { p + 1 , q + 1 } ^ { m , n + 1 } \left[A\left(\frac{x}{t}\right)^{\sigma}\right.\right. \\
& \left.\left.\begin{array}{r}
\left(g_{1}, G_{1} ; \in_{1}: y\right),(1-k, \sigma ; 1),\left(g_{j}, G_{j} ; \in_{j}\right)_{2, n},\left(g_{j}, G_{j}\right)_{n+1, p} \\
\left(h_{j}, H_{j}\right)_{1, m},(1-\lambda, \sigma ; 1),\left(h_{j}, H_{j} ; \mathcal{K}_{j}\right)_{m+1, q}
\end{array}\right] d x\right) d t
\end{aligned}
$$

Now, with the help of Equation (16), we get

$$
\varphi(\xi)=\frac{\Gamma(k-\xi)}{\sigma \Gamma(\lambda-\xi)} A^{-\frac{\xi}{\sigma}} \bar{f}\left(\frac{\xi}{\sigma}, y\right) \int_{0}^{\infty} t^{\xi-k} D^{\lambda-k}\{f(t)\} d t
$$

Further, applying the well known Mellin inversion theorem, we get

$$
D^{\lambda-k}\{f(t)\}=\frac{\sigma}{2 \pi i} \int_{\mathcal{L}} t^{k-\xi-1} \frac{\Gamma(\lambda-\xi)}{\Gamma(k-\xi)} A^{\frac{\xi}{\sigma}}\left[\bar{f}\left(\frac{\xi}{\sigma}, y\right)\right]^{-1} \varphi(\xi) d \xi
$$

Next, operating both sides by $D^{k-\lambda}$, we have

$$
f(t)=\frac{\sigma}{2 \pi i} D^{k-\lambda}\left\{\int_{\mathcal{L}} t^{k-\xi-1} \frac{\Gamma(\lambda-\xi)}{\Gamma(k-\xi)} A^{\frac{\xi}{\sigma}}\left[\bar{f}\left(\frac{\xi}{\sigma}, y\right)\right]^{-1} \varphi(\xi) d \xi\right\},
$$


which finally yields

$$
f(x)=\frac{\sigma x^{\lambda-1}}{2 \pi i} \int_{\mathcal{L}}\left[\bar{f}\left(\frac{\xi}{\sigma}, y\right)\right]^{-1} x^{-\xi} \varphi(\xi) d \xi
$$

Theorem 8. Let

(i) $m, n, p, q$ are nonnegative integers s.t. $1 \leq m \leq q$ and $0 \leq n \leq p$.

(ii) $\Re\left(k+\sigma\left(\frac{\mathrm{g}_{\mathrm{j}}-1}{\mathrm{G}_{\mathrm{j}}}\right)\right)<0 \quad(j=1, \cdots, n) ; \Re\left[k+\sigma\left(\frac{\mathrm{h}_{\mathrm{j}}}{\mathrm{H}_{\mathrm{j}}}\right)\right]>0 \quad(j=1, \cdots, m)$.

(iii) $-\min _{1 \leq j \leq m}\left(\frac{\Re\left(h_{j}\right)}{H_{j}}\right)<\Re(\xi)<\min _{1 \leq j \leq n}\left(\frac{\Re\left(1-g_{j}\right)}{G_{j}}\right)$.

(iv) $y \geq 0, \sigma>0, \lambda \in \mathbb{C}$ and $f, g \in \mathfrak{A}$.

Then, the following integral equation

$$
\int_{0}^{\infty} t^{-\lambda} \bar{\gamma}_{p, q}^{m, n}\left[A\left(\frac{x}{t}\right)^{\sigma} \mid \begin{array}{c}
\left(g_{1}, G_{1} ; \in_{1}: y\right),\left(g_{j}, G_{j} ; \epsilon_{j}\right)_{2, n},\left(g_{j}, G_{j}\right)_{n+1, p} \\
\left(h_{j}, H_{j}\right)_{1, m},\left(h_{j}, H_{j} ; \mathcal{K}_{j}\right)_{m+1, q}
\end{array}\right] f(t) d t=g(x)
$$

has a solution given by

$$
f(x)=\frac{\sigma x^{\lambda-1}}{2 \pi i} \int_{\mathcal{L}}\left[\bar{F}\left(\frac{\xi}{\sigma}, y\right)\right]^{-1} x^{-\xi} \varphi(\xi) d \xi
$$

where

$$
\varphi(\xi)=\int_{0}^{\infty} x^{\xi-1} g(x) d x
$$

and $\bar{F}(\xi, y)$ is given in Equation (14).

Proof. We here omit the details since the proof is parallel to that of Theorem 7.

\section{Applications}

In this section, we present some already existing results which directly follow from our main result. Next, we establish a few interesting results which are obtained from our main result by specializing the parameters of incomplete $\mathrm{H}$-functions and incomplete $\bar{H}$-functions.

(i) If we reduce IHF into Fox H-function in the assertions in Equations (20) and (22), then the result obtained by Srivastava and Raina follows [18].

(ii) If IHF is reduced into the Gauss hypergeometric function $\left.{ }_{2} F_{1}\right)$ in Equations (20) and (22), then the result collected by Prabhakar and Kashyap follows [17].

(iii) In Theorems 3 and 4, if IHFs are reduced into the incomplete Fox-Wright $\Psi$-functions ${ }_{P} \Psi_{Q}^{(\Gamma)}$ and ${ }_{P} \Psi_{Q}^{(\gamma)}$ (for details, see ([22] p.3, Equation (15))), then we get the solution of Fredholm integral equation involving the incomplete Fox-Wright $\Psi$-function ${ }_{P} \Psi_{Q}^{(\Gamma)}$ and ${ }_{P} \Psi_{Q}^{(\gamma)}$.

(iv) In Theorems 7 and 8, if IHFs are reduced into incomplete Fox-Wright $\bar{\Psi}$-functions $P \bar{\Psi}_{Q}^{(\Gamma)}$ and ${ }_{P} \bar{\Psi}_{Q}^{(\gamma)}$ (for details, see ([27] p.1216, Equation (1.7))), then we get the solution of Fredholm integral equation involving the incomplete Fox-Wright $\bar{\Psi}$-function ${ }_{P} \bar{\Psi}_{Q}^{(\Gamma)}$ and ${ }_{P} \bar{\Psi}_{Q}^{(\gamma)}$.

Our main results are general in nature; thus, by specializing the parameters of incomplete $\mathrm{H}$-functions and incomplete $\bar{H}$-functions, we obtain many Fredholm-type integral equations involving 
different types of functions in the kernel having various applications in fields including fluid mechanics, mathematical physics, quantum mechanics, queuing theory, etc. (for details, see [1-11]).

\section{Conclusions}

Our derived results are very important in diverse fields. We can obtain many results by setting specific values of different parameters of IHFs and incomplete $\bar{H}$-functions. In this work, we solve the Fredholm-type integral equation involving the incomplete $H$-function and incomplete $\bar{H}$-function in the kernel. Next, we obtain the Melin transform of incomplete $\bar{H}$-function. Further, we indicate some known results by setting specific values of different parameters of incomplete $\mathrm{H}$-function and incomplete $\bar{H}$-function. Thus, the results determined in this article would at once give numerous results involving a large variety of special functions appearing in different problems of science and engineering fields.

Author Contributions: Conceptualization, D.K.; Formal analysis, M.K.B., D.K., J.S. and K.S.N.; Funding acquisition, K.S.N.; Investigation, M.K.B.; Supervision, D.K.; Validation, J.S.; Writing-original draft, M.K.B. and J.S.; Writing-review and editing, D.K. and K.S.N. All authors have read and agreed to the published version of the manuscript.

Funding: This research supported by the TEQIP-III under CRS Grant 1-5730065311.

Conflicts of Interest: The authors declare no conflict of interest.

\section{References}

1. Treanţă, S. On the Kernel of a Polynomial of Scalar Derivations. Mathematics 2020, 8, 515. [CrossRef]

2. Treanţă, S. Gradient Structures Associated with a Polynomial Differential Equation. Mathematics 2020, 8, 535. [CrossRef]

3. Treanţă, S.; Vârsan, C. Weak small controls and approximations associated with controllable affine control systems. J. Differ. Equ. 2013, 255, 1867-1882. [CrossRef]

4. Doroftei, M.M.; Treanţă, S. Higher order hyperbolic equations involving a finite set of derivations. Balk. J. Geom. Appl. 2012, 17, 22-33.

5. Hu, S.; Khavanin, M.; Zhuang, W. Integral equations arising in the kinetic theory of gases. Appl. Anal. 1989, 34, 261-266. [CrossRef]

6. Manam, S.R. Multiple integral equations arising in the theory of water waves. Appl. Math. Lett. 2011, 24, 1369-1373. [CrossRef]

7. Koshev, A.; Kuzina, V. Solution of Integral Equations Arising in Mathematical Problems of Construction Science, using the Bogolyubov - Krylov method. IOP Conf. Ser. Mater. Sci. Eng. 2019, 603, 1-5. [CrossRef]

8. Ramm, A.G. Solution of Some Integral Equations Arising in Integral Geometry. Appl. Math. Lett. 1991, 4, 77-81. [CrossRef]

9. Ramm, A.G. Integral Equations Arising in the Open System Theory. In Theory and Applications of Some New Classes of Integral Equations; Springer: New York, NY, USA, 1980.

10. McDaniel, S.T.; Krauss, P.R. Expansion of integral equations arising in scattering theory. J. Acoust. Soc. Am. 1991, 89, 1113. [CrossRef]

11. Ioannou, Y.; Fyrillas, M.M.; Doumanidis, C. Approximate solution to Fredholm integral equations using linear regression and applications to heat and mass transfer. Eng. Anal. Bound. Elem. 2012, 36, 1278-1283. [CrossRef]

12. Buschman, R.G. An inversion integral. Proc. Am. Math. Soc. 1962, 13, 675-677. [CrossRef]

13. Erdéiyi, A. An integral equation involving Legendre functions. J. Soc. Ind. Appl. Math. 1964, 12, 15-30. [CrossRef]

14. Love, E.R. Some integral equations involving hypergeometric functions. Proc. Edinb. Math. Soc. 1967, 15, 169-198. [CrossRef]

15. Love, E.R.; Prabhakar, T.R.; Kashyap, N.K. A confluent hypergeometric integral equation. Glasg. Math. J. 1982, 23, 31-40. [CrossRef]

16. Prabhakar, T.R. A class of integral equations with Gauss functions in the kernels. Math. Nachr. 1972, 52, 71-83. [CrossRef] 
17. Prabhakar, T.R.; Kashyap, N.K. A new class of hypergeometric integral equations. Indian J. Pure Appl. Math. 1980, 11, 92-97.

18. Srivastava, H.M.; Raina, R.K. On certain methods of solving a class of integral equation of Fredholm type. J. Austral. Math. Soc. (Ser. A) 1992, 52,1-10. [CrossRef]

19. Srivastava, H.M.; Gupta, K.C.; Goyal, S.P. The H-Functions of One and Two Variables with Applications; South Asian Publishers: New Delhi, India, 1982.

20. Mathai, A.M.; Saxena, R.K. The H-Function with Applications in Statistics Other Disciplines; Wiley Eastern, New Delhi \& Wiley Halsted: New York, NY, USA, 1978.

21. Bansal, M.K.; Kumar, D.; Jain, R. Interrelationships Between Marichev-Saigo-Maeda Fractional Integral Operators, the Laplace Transform and the $\bar{H}$-Function. Int. J. Appl. Comput. Math. 2019, 5, 103. [CrossRef]

22. Bansal, M.K.; Choi, J. A Note on Pathway Fractional Integral Formulas Associated with the Incomplete $H$-functions. Int. J. Appl. Comput. Math. 2019, 5, 133. [CrossRef]

23. Bansal, M.K.; Kumar, D.; Nisar, K.S.; Singh, J. Application of incomplete H-functions in determination of Lambert's Law. J. Interdiscip. Math. 2019, 22, 1205-1212. [CrossRef]

24. Bansal, M.K.; Kumar, D.; Khan, I.; Singh, J.; Nisar, K.S. Certain Unified Integrals Associated with Product of $M$-Series and Incomplete $H$-functions. Mathematics 2019, 7, 1191. [CrossRef]

25. Bansal, M.K.; Kumar, D.; Nisar, K.S.; Singh, J. Certain fractional calculus and integral transform results of incomplete $\aleph$-functions with applications. Math. Meth. Appl. Sci. 2020, 43, 5602-5614. [CrossRef]

26. Srivastava, H.M.; Saxena, R.K.; Parmar, R.K. Some Families of the Incomplete $H$-functions and the Incomplete $\bar{H}$-functions and Associated Integral Transforms and Operators of Fractional Calculus with Applications. Russ. J. Math. Phys. 2018, 25, 116-138. [CrossRef]

27. Kumar, D.; Bansal, M.K.; Nisar, K.S.; Singh, J. Mathematical Modelling of Internal Blood Pressure Involving Incomplete $\bar{H}$-functions. J. Interdiscip. Math. 2019, 22, 1213-1221. [CrossRef]

28. Bansal, M.K.; Kumar, D. On the integral operators pertaining to a family of incomplete $I$-functions. AIMS-Math. 2020, 5, 1247-1259. [CrossRef]

29. Chaurasia, V.B.L.; Kumar, D. On the Solutions of Integral Equations of Fredholm type with Special Functions. Tamsui Oxf. J. Inf. Math. Sci. 2012, 28, 49-61.

30. Inayat-Hussain, A.A. New Properties of Hypergeometric Series Derivable from Feynman Integrals. II: A Generalisations of the H-Function. J. Phys. A 1987, 20, 4119-4128. [CrossRef]

31. Lighthill, M.J. Introduction to Fourier Analysis and Generalized Functions; Cambridge Monographs on Mechanics and Applied Mathematics; Cambridge University Press: Cambridge, UK; London, UK; New York, NY, USA, 1958.

32. Miller, K.S.; Ross, B. An Introduction to the Fractional Calculus and Fractional Differential Equations; Wiley-Interscience Publication, John Wiley and Sons: New York, NY, USA; Chichester, UK; Brisbane, Australia; Toronto, ON, Canada; Singapore, 1993. 